\title{
Épaisseur optique d'une couche de suie formée par une flamme de diffusion en micropesanteur
}

\author{
Guillaume Legros $^{1, a}$, Pierre Joulain $^{1}$, Jean-Pierre Vantelon ${ }^{1}$, Catherine Breillat ${ }^{1}$ \\ ET José TORERO ${ }^{2}$ \\ 1 Laboratoire de Combustion et de Détonique (UPR 9028 du CNRS), BP 40109, 86961 Futuroscope Cedex, France \\ 2 School of Engineering and Electronics, The University of Edinburgh, The King's Buildings, Edinburgh, EH9 3JN, UK
}

Reçu le 22 septembre 2003, accepté le 9 février 2004

\begin{abstract}
Résumé - Le besoin d'un critère d'inflammabilité des matériaux est avéré dès lors que la sécurité-incendie des engins spatiaux habités est envisagée. Différentes limites du nombre de transfert de masse apparaissent essentielles pour définir ce critère. Cependant, l'estimation de l'indice de confiance en ce critère requiert la connaissance de l'incertitude liée aux transferts radiatifs, encore mal connus en micropesanteur. Afin de découpler les phénomènes de pyrolyse et de dégagement de chaleur, une flamme non-prémélangée d'éthylène a été étudiée en micropesanteur. L'absorption radiative par la couche de suie formée représente la première étape de la caractérisation des phénomènes radiatifs. L'émission spontanée des radicaux $\mathrm{CH}^{*}$ précise alors la zone de réaction tridimensionnelle. Une mesure par extinction laser permet d'y corréler le champ d'absorption des suies. L'évolution spectrale de l'épaisseur optique de la couche de suie est ainsi déduite.
\end{abstract}

Mots clés : Flamme de diffusion / micropesanteur / transfert radiatif / suie / tomographie

Abstract - Optical thickness of a soot layer produced by a diffusion flame under microgravity conditions. Any fire safety study for manned spacecraft clearly shows that adequate criteria are required. A critical mass transfer number appears as an essential parameter to precise these criteria. Yet its validation relies on a deeper knowledge of the flame heat radiative transfer, still unclear for microgravity flames. In order to model a pyrolysing fuel, a non-premixed ethylene flame has been investigated in microgravity. The soot layer absorption is the first step towards radiative phenomena characterization. The tridimensionnal reaction zone is then located through $\mathrm{CH}^{*}$ radicals spontaneous emission. In summary, a laser extinction diagnostics allows to map the soot absorption field. The spectral optical thickness of the soot layer is consequently inferred.

Key words: Diffusion flame / microgravity / radiative transfer / soot / tomography

\section{Introduction}

Dès lors que la sécurité en terme d'incendie des engins spatiaux habités a sérieusement été étudiée, le besoin d'un critère d'inflammabilité et de l'indice de confiance qui l'accompagne s'est fait ressentir. Sur la base du test NASA 1 préexistant [1], Torero et al. [2] ont proposé une méthode plus précise pour aboutir au critère recherché à gravité normale.

Le nombre de transfert de masse $B$ apparaît comme le paramètre prépondérant, quantifiant en quelque sorte la propension de la flamme à se propager sur le matériau considéré. Cependant, la théorie d'Emmons [3]

\footnotetext{
a Auteur correspondant : legros@lcd.ensma.fr
}

sur laquelle repose l'élaboration du critère relève de plusieurs hypothèses, dont il s'agit de vérifier la validité. En particulier, Rouvreau et al. [4] ont montré, par une approche numérique, que sous certaines conditions aérodynamiques, la flamme reste en grande partie bidimensionnelle.

Poursuivant ces efforts de validation, la présente étude s'attaque à une hypothèse supplémentaire, consistant à négliger les propriétés réactives et radiatives des produits de combustion. Pour lever cette limitation, une redéfinition des termes de sources et de puits d'énergie ainsi que d'espèces s'avère nécessaire. On incorpore dans le nombre de transfert de masse différentes pertes enthalpiques $Q$ à la surface combustible, dont le flux en 


\section{Nomenclature}

\begin{tabular}{|c|c|c|}
\hline $\bar{a} a_{\lambda}$ & coefficient spectral local d'absorption & $\mathrm{m}^{-1}$ \\
\hline$A_{\lambda}$ & facteur spectral d'absorption & - \\
\hline$B$ & nombre de transfert de masse & - \\
\hline$f_{\text {suie }}$ & fraction volumique de suie & - \\
\hline$H$ & enthalpie & $\mathrm{J}$ \\
\hline height & hauteur & $\mathrm{m}$ \\
\hline$I_{\lambda}$ & intensité à la longueur d'onde $\lambda$ & unité arbitraire \\
\hline$k_{\lambda}$ & coefficient spectral local d'extinction & $\mathrm{m}^{-1}$ \\
\hline$K_{\lambda}$ & épaisseur optique à la longueur d'onde $\lambda$ & - \\
\hline$\stackrel{\bullet}{m}_{\mathrm{f}}^{\prime \prime}$ & débit surfacique de combustible pyrolysé & $\mathrm{kg} \cdot \mathrm{s}^{-1} \cdot \mathrm{m}^{-2}$ \\
\hline$n$ & partie réelle de l'indice de réfraction & - \\
\hline$\stackrel{\circ}{q}^{\prime \prime}$ & pertes enthalpiques surfaciques & J.m $\mathrm{m}^{-2}$ \\
\hline$Q$ & pertes enthalpiques & $\mathrm{J}$ \\
\hline$s$ & abscisse curviligne & $\mathrm{m}$ \\
\hline$S$ & signal mesuré & unité arbitraire \\
\hline width & épaisseur & $\mathrm{m}$ \\
\hline \multicolumn{3}{|c|}{ Symboles grecs } \\
\hline$\eta$ & coefficient de transmission des optiques & - \\
\hline$\kappa$ & partie imaginaire de l'indice de réfraction & - \\
\hline$\dot{\omega}$ & puissance volumique de réaction & $\mathrm{W} \cdot \mathrm{m}^{-3}$ \\
\hline \multicolumn{3}{|c|}{ Indices } \\
\hline cond & nature conductive & \\
\hline $\mathrm{f}$ & flamme & \\
\hline$\lambda$ & grandeur spectrale & \\
\hline $\mathrm{r}$ & nature radiative & \\
\hline $\mathrm{s}$ & surface combustible & \\
\hline side & vue de côté & \\
\hline top & vue de dessus & \\
\hline
\end{tabular}

retour à la surface rayonné par la flamme $\stackrel{\circ}{\mathrm{f}, \mathrm{r}}^{\prime \prime}$, ajouté comme un terme négatif :

$$
\dot{m}_{\mathrm{f}}^{\prime \prime} Q=\stackrel{\leftrightarrow}{q}_{\text {cond }}^{\prime \prime}+\stackrel{\bullet}{q}_{\mathrm{s}, \mathrm{r}}^{\prime \prime}-\stackrel{\circ}{\mathrm{f}, \mathrm{r}}^{\prime \prime}
$$

Dès lors que les pertes par conduction à travers l'épaisseur du combustible $\stackrel{q}{\text { cond }}_{\text {"I }}$ ont, par le passé, été étudiées [2] et qu'un certain nombre de données permettent d'ores et déjà d'estimer précisément l'émission $\dot{q}_{\mathrm{s}, \mathrm{r}}^{\prime \prime}$ de la surface, il apparaît fondamental d'évaluer la transmission du flux radiatif en retour à la surface au travers de la couche de suie. Ainsi, la précision du calcul du nombre de transfert de masse sera-t-elle accrue et, par là-même, celle du débit de combustible pyrolysé $\dot{m}_{\mathrm{f}}^{\prime \prime}$, ce dernier étant proportionnel à $B$.

L'ensemble des résultats expérimentaux ici exposés ont été obtenus au cours de campagnes de vols paraboliques du CNES et de l'ESA. Modélisant à nouveau la zone de pyrolyse grâce à une injection de combustible gazeux, une flamme d'éthylène, établie au sein d'une couche-limite sur un brûleur plat, est alimentée par un débit transversal d'oxydant. Afin d'évaluer les effets tridimensionnels de la zone de réaction, cette étude a eu recours à une mesure de l'émission spontanée des radicaux $\mathrm{CH}^{*}$. Une technique d'extinction laser a été mise en œuvre pour obtenir un champ d'extinction à travers la flamme. L'épaisseur optique de la couche de suie en est déduite sur l'ensemble $\mathrm{du}$ spectre du transfert thermique radiatif. Ainsi, une méthodologie pour évaluer plus généralement le rôle absorbant des suies est-elle proposée.

\section{Mise en œuvre expérimentale}

La flamme non-prémélangée est établie sur un brûleur gaz-gaz, dont le schéma de la figure 1 donne les dimensions. Le poreux, placé de sorte à ne pas entraver la planéité de la plaque, permet l'injection uniforme d'éthylène sur une section efficace de $50 \mathrm{~mm} \times 50 \mathrm{~mm}$. L'écoulement transversal d'oxydant est laminarisé à travers d'un nid d'abeilles situé à l'entrée de la chambre de combustion.

L'alimentation en combustible et en oxydant est régulée par des débitmètres massiques. Le brûleur est installé au sein d'une chambre de combustion, dont l'évacuation est régulée par une électrovanne, qui conditionne 


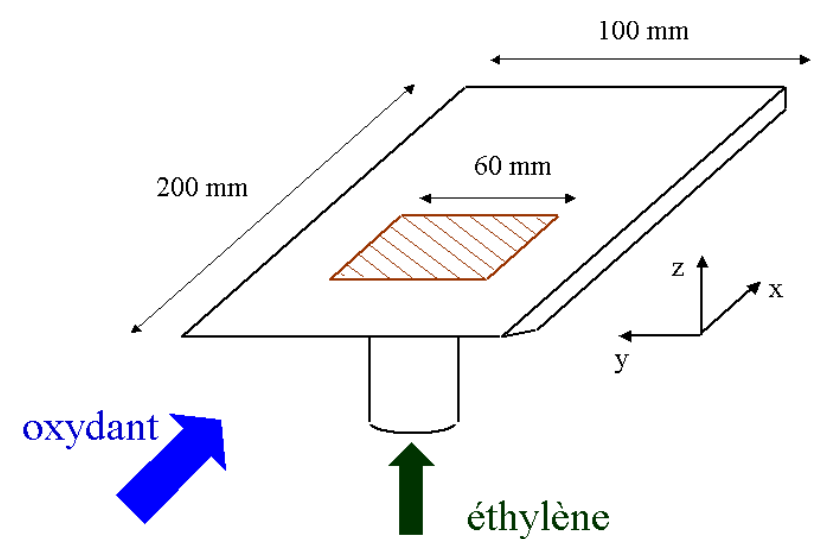

Fig. 1. Schéma du brûleur.

la chambre à la pression atmosphérique. Par ailleurs, la chambre est munie d'un ensemble de hublots permettant différentes visualisations.

Afin d'obtenir une recomposition tridimensionnelle de la zone réactive, deux caméras numériques à matrice CCD Sony DCR-TVR17E ont été installées (cf. Fig. 2) : l'une à l'aplomb du poreux, l'autre sur le côté. Chacune d'elles a été munie d'un filtre passe-bande Melle \& Griot centré sur la longueur d'onde $\lambda=431 \mathrm{~nm}$ et de largeur à mihauteur de $10 \mathrm{~nm}$. La première caméra filme la flamme en vue de dessus, alors que la seconde retransmet des profils de cette même flamme (cf. Fig. 2).

La mesure d'extinction utilise ici une diode laser verte, émettant à la longueur d'onde $\lambda=532 \mathrm{~nm}$. Sa divergence naturelle permet de couvrir l'ensemble du domaine d'étude lorsque la diode est tenue suffisamment loin de cette zone. Après avoir traversé la flamme (i.e. selon l'axe $y$ ), le faisceau est réfléchi à $60 \%$ par une lame mince avant d'impresionner la matrice CCD d'une caméra numérique, munie, elle aussi, d'un filtre passe-bande, de largeur $10 \mathrm{~nm}$ à mi-hauteur mais centré cette fois sur $532 \mathrm{~nm}$. Enfin, un obturateur rotatif relativement lent vient alternativement éteindre l'émission laser de sorte que la caméra enregistre approximativement 10 images d'extinction consécutives à 10 autres images d'émission verte naturelle. La tache centrale créée par la diode laser dans l'objectif de la caméra est alors centrée sur le domaine d'étude, à savoir juste au-dessus du poreux. De cette façon, les rayons provenant de la diode sont considérés comme étant parallèles entre eux et orthogonaux au plan de mesure de l'extinction. Dans cette zone, il est alors permis de parler de mesure d'extinction ou, plus précisément, d'épaisseur optique le long de l'axe $y$. Il s'agit alors de revenir à l'épaisseur optique le long de l'axe $z$ pour quantifier le pouvoir absorbant de la couche de suie face au flux radiatif émis par la flamme.

La synchronisation de l'ensemble de ces visualisations est réalisée par diodes bleues et vertes, qui s'éteignent lorsque débute le test.

L'étude présentée ici ayant pour objectif l'évaluation du pouvoir absorbant des suies, seuls les résultats issus d'un couple de vitesse sont exposés. Il s'agit en l'occurence d'une vitesse de soufflage de $150 \mathrm{~mm} . \mathrm{s}^{-1}$ et d'une vitesse d'injection de $5 \mathrm{~mm} . \mathrm{s}^{-1}$. Le combustible choisi est l'éthylène, du fait de sa propension à produire des suies. Le mélange oxydant est, quant à lui, composé à $65 \%$ en $\mathrm{N}_{2}$ et à $35 \%$ en $\mathrm{O}_{2}$. En effet, un taux en dioxygène relativement élevé permet à la fois de favoriser localement la production en suie et d'obtenir des niveaux acceptables d'intensité par chimiluminescence des radicaux $\mathrm{CH}^{*}$.

\section{Principe général de la tomographie}

Des mesures d'extinction d'une source laser permettent de remonter à l'épaisseur optique spectrale $K_{\lambda}(s)$, grandeur qui quantifie la proportion absorbée d'une émission à la longueur d'onde considérée $\lambda$ le long d'un chemin allant de 0 à $s$. Ainsi la loi de Bouguer s'écritelle comme suit :

$$
\frac{I_{\lambda}(s)}{I_{\lambda}(0)}=e^{-K_{\lambda}(s)}
$$

avec $I_{\lambda}(0)$ l'intensité de la source à l'entrée du milieu étudié, $I_{\lambda}(s)$ l'intensité de la source à une abscisse quelconque du milieu étudié et

$$
K_{\lambda}(s)=\int_{0}^{s} k_{\lambda}\left(s^{\prime}\right) \mathrm{d} s^{\prime}
$$

où $k_{\lambda}\left(s^{\prime}\right)$ est le coefficient local d'extinction, sachant que ce terme d'extinction est la somme d'un terme de diffusion et d'un terme d'absorption.

Si la diffusion peut être négligée face à l'absorption, la loi de Bouguer se réduit à l'équation ci-dessous, où $a_{\lambda}\left(s^{\prime}\right)$ est le coefficient local d'absorption :

$$
\frac{I_{\lambda}(s)}{I_{\lambda}(0)}=e^{-\int_{0}^{s} a_{\lambda}\left(s^{\prime}\right) \mathrm{d} s^{\prime}}=e^{-A_{\lambda}(s)}
$$

Le coefficient $A_{\lambda}(s)$, résultat de l'intégration de $a_{\lambda}(s)$ le long du chemin optique, correspond alors à la mesure d'extinction effective :

$$
A_{\lambda}(s)=-\ln \left(\frac{I_{\lambda}(s)}{I_{\lambda}(0)}\right)
$$

Il est important de noter ici que cette mesure $\frac{I_{\lambda}(s)}{I_{\lambda}(0)}$ de l'extinction de la source peut engendrer une erreur conséquente dès lors que cette source induit une émission du milieu le long de son trajet. Pour autant, Siegel et Hottel [5] montrent que cette erreur ne saurait excéder $1 \%$ de la mesure dans la mesure où le produit $\lambda T$ est inférieur à $3120 \mu \mathrm{m} . \mathrm{K}$. À la longueur d'onde expérimentale de $532 \mathrm{~nm}$, atteindre une erreur de $1 \%$ impliquerait des températures de l'ordre de $5865 \mathrm{~K}$, ce qui est à l'évidence hors du domaine des températures atteintes par une flamme de diffusion peu ou pas confinée. En conséquence, la présente étude ne prend aucune considération de l'émission induite par la source de la tomographie. 


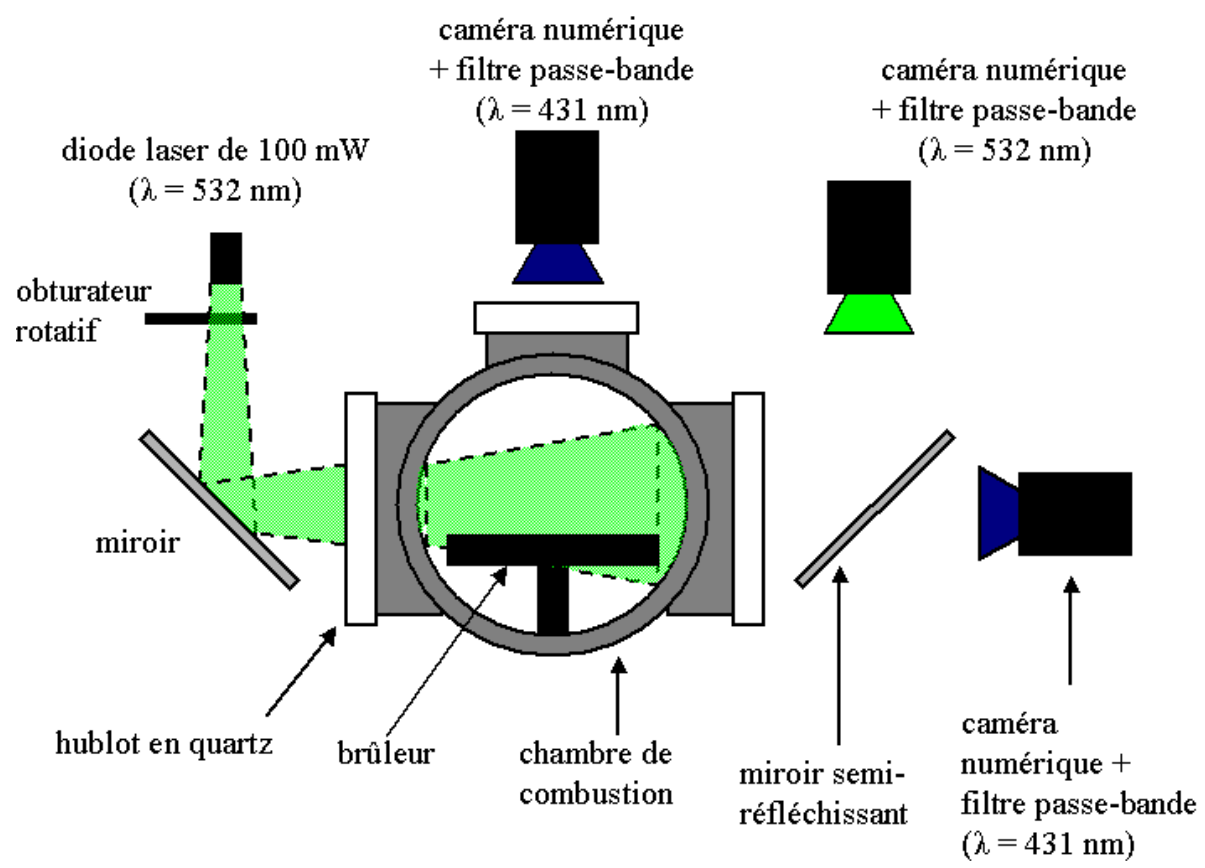

Fig. 2. Schéma du montage expérimental vu depuis l'entrée du flux oxydant.

En revanche, il apparaît nécessaire de prendre en compte l'émission spontanée du milieu, afin de la soustraire aux mesures d'intensité. Il s'agit alors de pulser la source et d'obtenir ainsi des images « éclairées » suivies d'images «non-éclairées », que nous qualifierons par la suite de «noires » par abus de langage. Les dernières seront soustraites des premières pour ne pas entacher la mesure par l'émission spontanée de la zone de réaction et de celle des suies.

Dans de telles conditions, Dalzell et Sarofim [6] montrent qu'une mesure unique d'extinction à une longueur d'onde donnée permet de passer de l'évolution spatiale du coefficient $a_{\lambda}$ à celle de la fraction volumique de suie par l'intermédiaire de la théorie de Mie :

$$
\frac{a_{\lambda}(s)}{f_{\text {suie }}}=\frac{36 n^{2} \kappa \pi}{\left(n^{2}\left(1-\kappa^{2}\right)+2\right)^{2}+4 n^{4} \kappa^{2}} \frac{1}{\lambda}
$$

où $n$ et $n \kappa$ sont respectivement les parties réelle et imaginaire de l'indice de réfraction du milieu.

De cette façon, une fraction volumique $f_{\text {suie }}$ de suie « absorbante » peut être étalonnée. L'évolution spatiale de cette fraction volumique le long de l'axe $z$ est alors déduite. En revenant enfin à l'équation (6), l'évolution de $a_{\lambda}$ donc de $K_{\lambda}(s)$ en fonction de $\lambda$ est connue. Le pouvoir absorbant de la couche de suie sur l'ensemble du spectre du transfert radiatif est alors quantifié.

\section{Principe de la correction de la tomographie}

On se propose ici de calquer la structure de la zone de présence des suies donnée par la tomographie sur celle de la zone de réaction révélée par les radicaux $\mathrm{CH}^{*}$ et reconstituée en trois dimensions.

\subsection{Chimiluminescence des radicaux $\mathrm{CH}^{*}$}

Un nombre important de travaux (par exemple $[7,8]$ ) considèrent les radicaux $\mathrm{CH}^{*}$ comme des acteurs majeurs évoluant au coeur même du schéma cinétique de la combustion des hydrocarbures. Ces radicaux, à la durée de vie extrêmement courte, sont les produits de la réaction entre les espèces $\mathrm{O}_{2}$ et $\mathrm{C}_{2} \mathrm{H}$ :

$$
\mathrm{O}_{2}+\mathrm{C}_{2} \mathrm{H} \longrightarrow \mathrm{CO}_{2}+\mathrm{CH}^{*}
$$

Ces radicaux excités retournent à leur état fondamental, soit par relaxation collisionnelle, soit par fluorescence spontanée, dont la transition, notée $A^{2} \Delta \rightarrow X^{2} \Pi$, est révélée à la longueur d'onde $\lambda=431,4 \mathrm{~nm}$.

En conséquence, certains auteurs ont relié directement des mesures de la chimiluminescence des $\mathrm{CH}^{*}$ au taux de combustion volumique [9-11]. Partant des travaux antérieurs de Hurle [12], McManus et al. [13] ont en effet supposé que la puissance volumique libérée $\dot{\omega}$ par la réaction était proportionnelle à l'intensité $I$ délivrée par la chimiluminescence des $\mathrm{CH}^{*}$ :

$$
\dot{\omega}=\beta I
$$

Il s'agit par la suite de définir le paramètre $\beta$ pour aboutir à des résultats quantitatifs. Ces travaux, menés sur des flammes de prémélange, mettent alors en œuvre un bilan d'enthalpie $\Delta H$, défini en soustrayant l'enthalpie sortante des produits de réaction à l'enthalpie entrante des réactifs. En intégrant ensuite l'intensité des $\mathrm{CH}^{*}$ sur l'ensemble du domaine d'étude $V, \beta$ peut être calculé par la relation suivante :

$$
\beta=\frac{\Delta H}{\int_{V} I \mathrm{~d} V}
$$


Dès lors, des résultats quantitatifs peuvent en effet être obtenus pour des flammes prémélangées, au sein desquelles le coefficient local d'équivalence est un paramètre ajustable. Les différentes concentrations des réactifs entrant dans la zone réactive sont alors précisément connues et un analyseur chimique permet de déterminer les concentrations en réactifs à la sortie.

Trois difficultés font alors entrave à l'application de cette technique à la présente étude.

\subsubsection{Application aux flammes de diffusion}

D'une part, une certaine prudence est requise quant à l'extrapolation de cette méthode aux flammes de diffusion. Néanmoins, certains résultats laissent à penser que cette extrapolation est justifiable. En particulier Berg [10] et Higgins [9] remarquent que la chimiluminescence des $\mathrm{CH}^{*}$ augmente lorsque l'on approche les conditions expérimentales de la stœechiométrie. Cette tendance est d'autant plus notable que la pression ambiante converge vers la pression atmosphérique. Ces éléments contribuent alors à la relative confiance en l'application de la technique à la flamme de diffusion établie en micropesanteur. En effet, le rapport du signal au bruit doit être élevé. Qui plus est, la pression étant ici la pression atmosphérique, la zone réactive est étroite et caractérisée par un rapport de mélange proche de la stoechiométrie.

\subsubsection{Aspect quantitatif de l'application}

D'autre part, le bilan d'enthalpie requiert une mise en œuvre relativement lourde en terme de volume du fait de la nécessité d'utiliser un analyseur chimique. Or, comme précisé plus avant, le volume est un paramètre limitant des expériences à bord de l'avion A300 zéro-G. En conséquence, ce bilan n'a pu être entrepris. Cependant, on peut remarquer que le paramètre $\beta$ devrait être constant pour des conditions expérimentales fixées. Il est ainsi possible de considérer l'évolution de l'intensité locale de la chimiluminescence par rapport à une valeur maximale mesurée.

\subsubsection{Bruitage du signal}

Enfin, les études citées plus avant se plaçaient dans le cas idéal d'un combustible produisant peu de suies. Malgré cela, Blevins et al. [11] jugent nécessaire d'appliquer au signal une correction afin de soustraire à l'intensité de la chimiluminescence une partie de l'émission considérée comme provenant de l'oxydation des suies. Toutefois, la concentration en suies est ici l'objet principal de l'étude. De fait, aucune correction à l'encontre de leur oxydation n'a été effectuée, de sorte que l'ensemble de la zone réactive a été étudiée.

\subsection{Reconstruction de la zone réactive par chimiluminescence}

Partant de ces remarques, il est possible de reconstruire en trois dimensions la zone de réaction à partir de deux vues bidimensionnelle orthogonales. Par référence au jet oxydant, la coordonnée longitudinale $x$ (cf. Fig. 1) est ici une variable du problème, notée en indice de chaque grandeur considérée pour rappeler la dépendance en $x$ de cette grandeur.

Considérons une mesure en vue latérale de la flamme par une matrice CCD, c'est-à-dire une vue dont la direction est parallèle à l'axe $y$ et qui intègre, par conséquent, sur chacun des pixels de la matrice, l'intensité $I(x, y, z)$

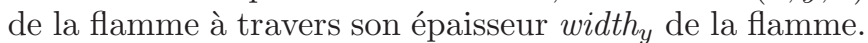
Ce signal $S_{x}(z)$, fonction de la localisation $(x, z)$ du pixel, est a priori proportionnel à l'angle solide $\Omega_{\text {side }}$ dans lequel est vue la flamme, au coefficient de transmission des optiques intermédiaires $\eta_{\text {side }}$, ainsi qu'à l'intensité intégrée le long du chemin optique :

$$
S_{x}(z)=\frac{\Omega_{\text {side }}}{4 \pi} \eta_{\text {side }} \int_{\text {width }_{y}} I(x, y, z) \mathrm{d} y
$$

De la même façon, une vue de dessus permet d'obtenir le signal $S_{x}(y)$, intègrant cette fois l'intensité de la flamme à travers l'épaisseur height :

$$
S_{x}(y)=\frac{\Omega_{\mathrm{top}}}{4 \pi} \eta_{\mathrm{top}} \int_{\text {height }_{z}} I(x, y, z) \mathrm{d} z
$$

Ici, l'indice « top » marque l'appartenance des propriétés à la vue de dessus.

Les équations (10) et (11) impliquent alors l'égalité suivante :

$$
\frac{\int_{\text {height }_{z}} S_{x}(z) \mathrm{d} z}{\int_{\text {width }_{y}} S_{x}(y) \mathrm{d} y}=\frac{\Omega_{\text {side }}}{\Omega_{\mathrm{top}}} \frac{\eta_{\text {side }}}{\eta_{\text {top }}}
$$

Cette dernière égalité montre que le membre de gauche de l'équation devrait être une constante à conditions expérimentales fixées. Une fois cette constante expérimentalement définie, l'égalité (12) doit permettre de déduire une des deux épaisseurs par la connaissance expérimentale de l'autre. Dans la démarche qu'adopte cette étude, il apparaît alors judicieux de considérer que l'épaisseur d'intégration selon l'axe $z$ height est bien moins la proie des phénomènes tridimensionnels que l'épaisseur d'intégration selon l'axe y widthy. En conséquence, c'est la vue de dessus qui intègre l'intensité sur l'épaisseur height $t_{z}$ qui servira d'étalon à la correction apportée sur les épaisseurs.

Par analogie, la correction sera ensuite extrapolée à l'épaisseur d'intégration permettant de définir l'épaisseur optique dans l'équation (3). 


\section{Détail de l'analyse tomographique proposée}

Avant de synthétiser l'ensemble des résultats apportés par la méthode proposée, il est préférable d'exposer dans le détail l'ensemble des étapes composant la dite méthode. Pour ce faire, le dépouillement d'un test est réalisé cidessous. La vitesse d'injection en éthylène est fixée à $5 \mathrm{~mm} . \mathrm{s}^{-1}$ tandis que celle du soufflage en oxydant est fixée à $150 \mathrm{~mm} \cdot \mathrm{s}^{-1}$. Ce mélange oxydant est constitué à $35 \%$ en $\mathrm{O}_{2}$ et à $65 \%$ en $\mathrm{N}_{2}$. Un taux ainsi enrichi en oxygène permet en effet d'augmenter localement la température de flamme, ce qui a pour effet de faciliter le craquage des molécules d'éthylène et d'augmenter en conséquence localement la production de suie. Qui plus est, le rapport signal/bruit émis par les radicaux $\mathrm{CH}^{*}$ est d'autant meilleur.

\subsection{Symétrie du problème}

L'origine du repère cartésien lié au domaine d'étude se trouve à l'intersection du bord amont de la section efficace du poreux et du plan de symétrie $(x, z)$ du brûleur. Ainsi le poreux est défini par le domaine $\{0 \leq x \leq 5 \mathrm{~mm}$; $-2,5 \mathrm{~mm} \leq y \leq 2,5 \mathrm{~mm} ; z=0 \mathrm{~mm}\}$. Les courbes expérimentales montrant cette symétrie, toute courbe fonction de $y$ sera uniquement représentée pour $y \leq 0$. De plus, le terme de «largeur »d'une courbe est à entendre par la suite comme largeur à mi-hauteur.

\subsection{Structure de la zone réactive}

Les figures 3 et 4 exposent les graphes de coupes de la zone réactive pour différentes coordonnées longitudinales $x$. L'unité, arbitraire, est fonction de l'exposition, de la caméra considérée. Il est évidemment ardu voire illusoire de régler les deux caméras au même seuil d'exposition, vu le nombre et la nature des optiques intermédiaires. Chacune des deux caméras possède ainsi sa propre échelle arbitraire d'intensité. De ces échelles dépend, en conséquence, la constante définie dans l'équation (12).

Le graphe de la figure 3 montre l'évolution en fonction de la coordonnée transverse $y$ de l'intensité de la chimiluminescence des $\mathrm{CH}^{*}$ tandis que le graphe de la figure 4 décrit, lui, l'évolution en fonction de la coordonnée verticale $z$ de cette même intensité. On constate ici que le maximum dans chacune des vues est atteint au mileu du poreux (i.e. pour $x=25 \mathrm{~mm}$ ). De plus, le profil gaussien des courbes en vue latérale tend à perdre sa symétrie à mesure que l'on s'éloigne du bord d'attaque du poreux. La modification de l'épaisseur d'intégration prend ici toute sa justification.

En référence à l'équation (12), la figure 5 établit l'évolution du rapport des intensités intégrées en fonction de la coordonnée longitudinale $x$. Une valeur effectivement constante de 0,08 se révèle dans un domaine $15 \mathrm{~mm} \leq x \leq 50 \mathrm{~mm}$ excluant le bord d'attaque et le

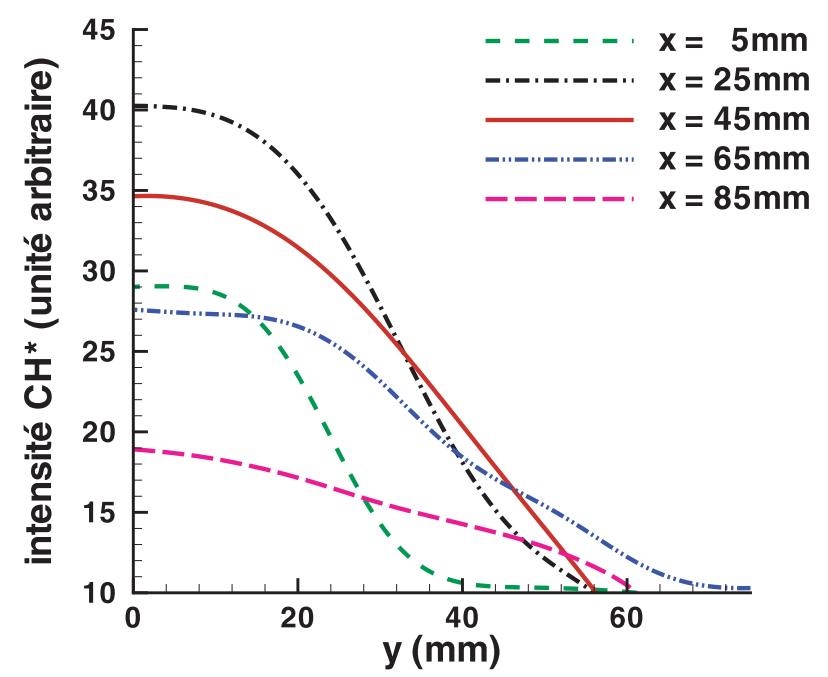

Fig. 3. Coupes en vue de dessus de l'intensité de l'émission spontanée des $\mathrm{CH}^{*}$ à différentes abscisses $x$.

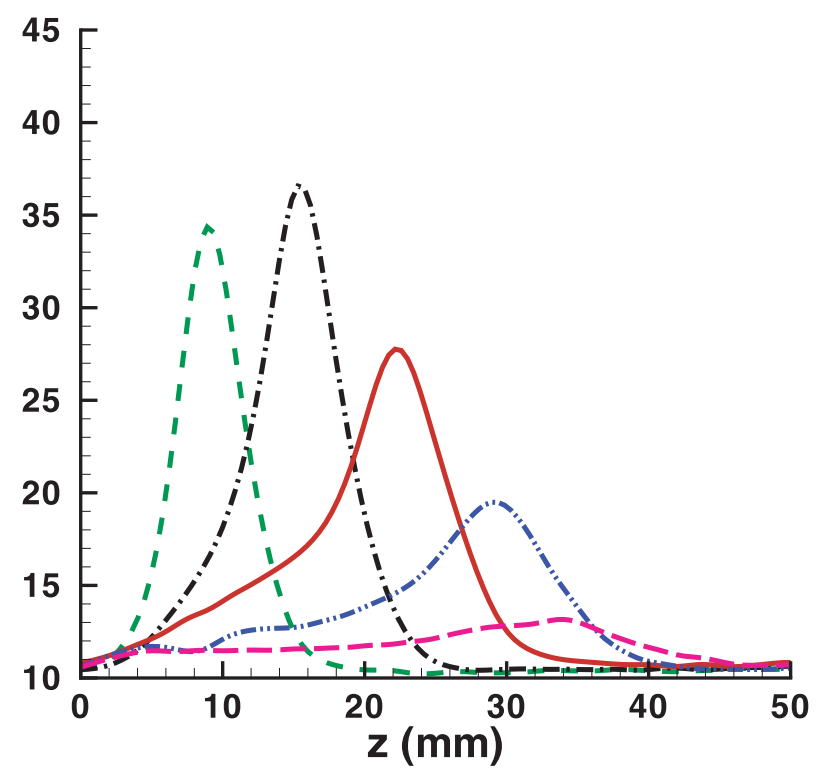

Fig. 4. Coupes en vue de côté de l'intensité de l'émission spontanée des $\mathrm{CH}^{*}$ à différentes abscisses $x$.

bord de fuite de la flamme. Une si faible valeur est la conséquence de deux faits :

- le signal $S_{x}(z)$ est transmis à travers le miroir semiréfléchissant $\left(\eta_{\text {side }}\right.$ petit devant $\left.\eta_{\text {top }}\right)$;

- la caméra placée pour la vue latérale est située plus loin que la caméra donnant la vue de dessus $\left(\Omega_{\text {side }}\right.$ petit devant $\left.\Omega_{\text {top }}\right)$.

En outre, le bord d'attaque et le bord de fuite de la flamme sont le siège de phénomènes toujours difficiles à identifier. En particulier, le bord de fuite est soumis à des phénomènes instationnaires tridimensionnels relativement rapides. Il est donc fort probable que la sensibilité des caméras, dont la fréquence d'acquisition est de 25 images à la seconde, ne permette pas de discerner ces phénomènes. 


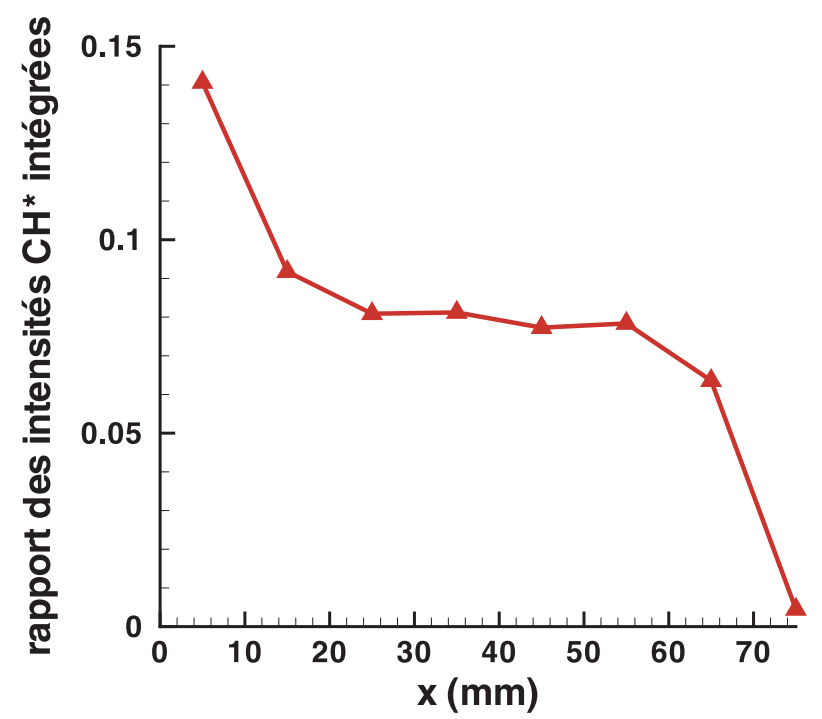

Fig. 5. Rapport des intensités $\mathrm{CH}^{*}$ intégrées (cf. Éq. (12)) fonction de l'abscisse $x$.

\subsection{Correction par analyse de la zone réactive}

Le signal $S_{x}(z)$, dont l'évolution est donnée sur la graphe de la figure 4 , peut être assimilé à une gaussienne de largeur $\Delta z$ et de hauteur $\operatorname{Max}_{z}$. De même, le signal $S_{x}(y)$, dont l'évolution est tracée sur la graphe de la figure 3, est approximativement la somme d'une gaussienne de largeur $\Delta y$ et de hauteur $M a x_{y}$ et d'une fonction créneau de largeur $50 \mathrm{~mm}$ (i.e. la largeur du poreux) et de même hauteur $\operatorname{Max}_{y}$.

Notons $\alpha$ la constante définie par le terme de droite de l'équation (12). L'équation (12) s'écrit alors comme suit :

$$
\frac{\sqrt{\frac{\pi}{\ln 2}} \frac{\Delta z \operatorname{Max}_{z}}{2}}{\sqrt{\frac{\pi}{\ln 2}} \frac{\Delta y \operatorname{Max}_{y}}{2}+50 \operatorname{Max}_{y}}=\alpha
$$

ce qui donne une expression à $\Delta z$ :

$$
\Delta z=\alpha \Delta y+100 \alpha \sqrt{\frac{\ln 2}{\pi}} \operatorname{Max}_{y}
$$

Cette équation montre que la largeur $\Delta z$ observée en vue latérale est due à la contribution d'une partie bidimensionnelle de la flamme et d'une partie $\Delta y$ tridimensionnelle de celle-ci.

De fait, la vue latérale peut être corrigée, en l'occurence de $8 \%$ ici, selon l'égalité suivante :

$$
\Delta z_{\text {corr }}=\Delta z-\alpha \Delta y
$$

Par analogie, cette correction sera apportée aux courbes d'extinction.

\subsection{Structure de la zone de suie}

La figure 6 est une image instantanée enregistrée par la matrice $\mathrm{CCD}$ de la caméra numérique chargée de la

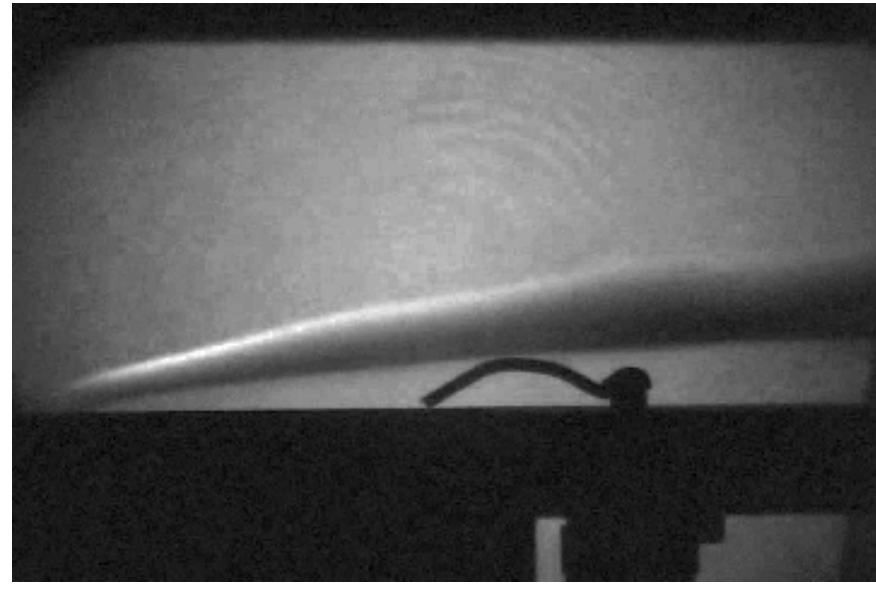

Fig. 6. Image instantanée de la tomographie laser pour une vitesse de soufflage oxydant de $150 \mathrm{~mm} . \mathrm{s}^{-1}$ et une vitesse d'injection en éthylène de $5 \mathrm{~mm} . \mathrm{s}^{-1}$.

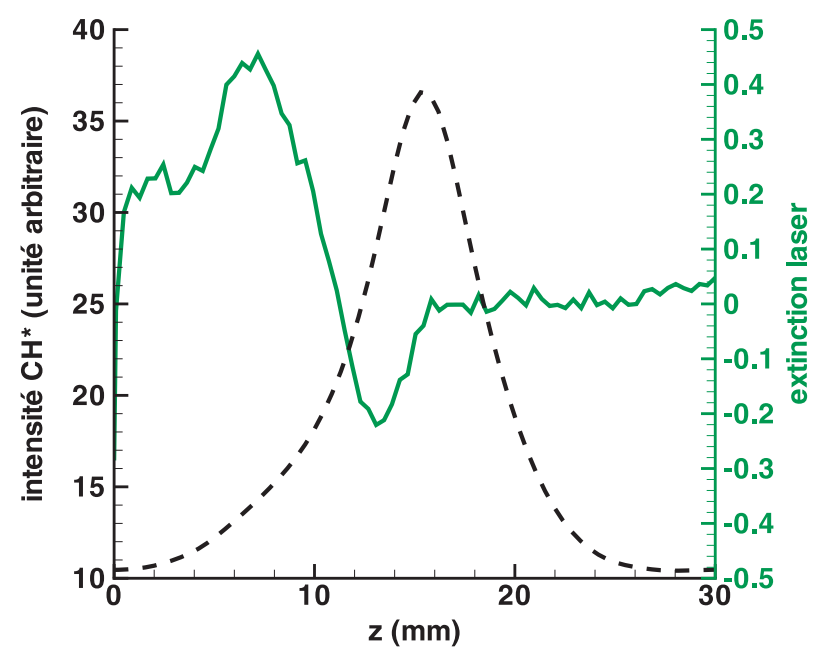

Fig. 7. Comparaison des évolutions de l'extinction (en trait plein) et de l'émission des $\mathrm{CH}^{*}$ (en pointillés), fonction de $z$ à $x=25 \mathrm{~mm}$.

mesure d'extinction. On remarque sur cette image que la couche de suie, placée juste sous la flamme (en surbrillance), s'épaissit au fur et à mesure que $x$ augmente.

De la même façon que pour l'intensité de la chimiluminescence des radicaux $\mathrm{CH}^{*}$, on peut tracer l'évolution de la mesure d'extinction $-\ln \left(\frac{I_{\lambda}(s)}{I_{\lambda}(0)}\right)$ en fonction de la coordonnée verticale $z$.

La figure 7 permet la comparaison entre les évolutions à $x$ donnée de l'intensité des $\mathrm{CH}^{*}$ et de l'extinction. Dans le cas présent, l'émission spontanée verte du milieu n'a pas été soustraite à l'intensité des images éclairées. L'interaction entre suies et radicaux $\mathrm{CH}^{*}$ est alors traduite par le recouvrement entre le pic négatif d'extinction (révélant la composante verte de la flamme) pour $x=13 \mathrm{~mm}$ et le pic de l'intensité des $\mathrm{CH}^{*}$ en $x=14,5 \mathrm{~mm}$. Le maximum d'émission verte, attribuée aux suies, est effectivement entre ces dernières et la zone de réaction, attribuée aux radicaux $\mathrm{CH}^{*}$. 


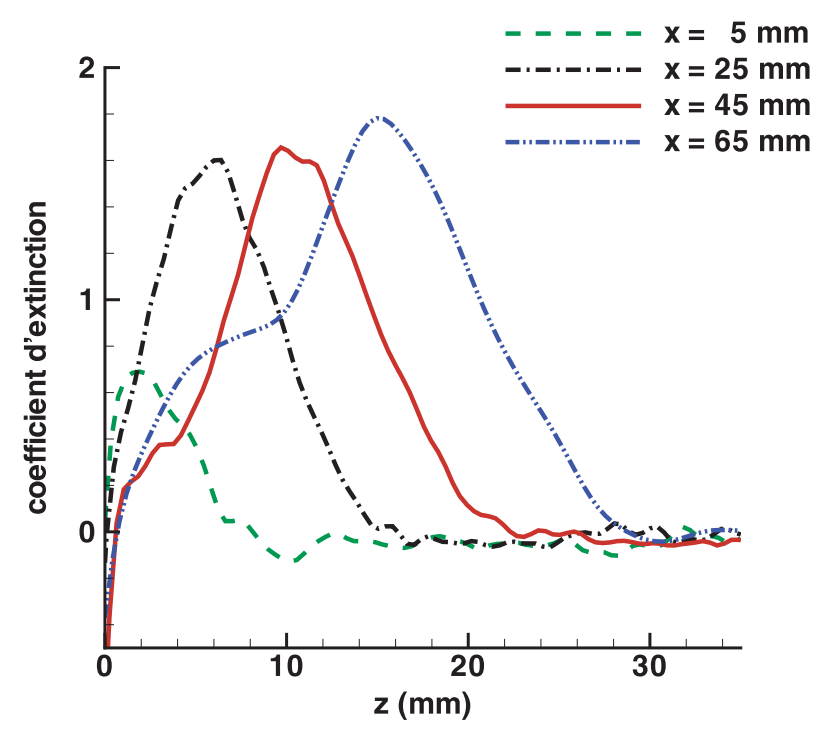

Fig. 8. Évolution du coefficient d'extinction en fonction de la coordonnée verticale $z$ pour différentes valeurs de la coordonnée longitudinale $x$.

La figure 8 expose, pour différentes valeurs de la coordonnée longitudinale $x$, l'évolution le long de la verticale du coefficient d'extinction $A_{\lambda}$ défini dans l'équation (5). À nouveau, on constate que le bord d'attaque (ici en $x=5 \mathrm{~mm}$ ) et le bord de fuite (en $x=65 \mathrm{~mm}$ ) donnent lieu à des comportements relativement insconstants. En revanche, le domaine au sein duquel l'intensité des radicaux $\mathrm{CH}^{*}$ montrait une allure constante, i.e. pour $15 \mathrm{~mm} \leq x \leq 50 \mathrm{~mm}$ (cf. Fig. 5), est le siège d'un comportement lui aussi constant du coefficient d'extinction, à la fois en amplitude et en largeur.

En supposant que la structure des suies est suffisamment constante dans ce domaine pour ne pas apporter de modification au coefficient d'absorption local, la zone considérée serait un volume où l'oxydation des suies compense leur formation, de sorte que leur concentration reste relativement stable. Cette remarque est par ailleurs en accord avec la stabilité de la constante $\alpha$ définie à partir de l'intensité des radicaux $\mathrm{CH}^{*}$.

L'originalité de la méthode décrite ici consiste à extrapoler la correction apportée à l'épaisseur $\Delta z$ de la zone de présence des radicaux $\mathrm{CH}^{*}$ en vue latérale à la correction à donner aux profils d'extinction, eux-mêmes issus d'une vue latérale. Cette opération consiste alors à considérer que le signal initial a traversé la large partie bidimensionnelle de la flamme, i.e. entre $-25 \mathrm{~mm} \leq y \leq 25 \mathrm{~mm}$ et que seule la largeur de la courbe du coefficient d'extinction est affectée par les effets de bord tridimensionnels. On constate à ce propos sur le graphe de la figure 8 que l'aile des formes gaussiennes s'élargit entre l'origine et le pic d'extinction.

\section{5 Étalonnage de la fraction volumique de suies}

Dès lors, les valeurs du coefficient spectral d'extinction peuvent être étalonnées en terme de fraction volumique
Tableau 1. Données intrapolées de l'éthylène (en gras) à partir des données expérimentales de Dalzell et Sarofim [6].

\begin{tabular}{ccccccc}
\hline $\begin{array}{c}\text { espèce } \\
\text { formule }\end{array}$ & \multicolumn{2}{c}{ acétylène } & \multicolumn{2}{c}{ éthylène } & \multicolumn{2}{c}{ propane } \\
$\mathrm{C}_{2} \mathrm{H}_{2}$ & \multicolumn{2}{c}{$\mathrm{C}_{2} \mathrm{H}_{4}$} & \multicolumn{2}{c}{$\mathrm{C}_{3} \mathrm{H}_{8}$} \\
$\mathrm{H} / \mathrm{C}$ & \multicolumn{2}{c}{1} & \multicolumn{2}{c}{2} & \multicolumn{2}{c}{2,67} \\
$\lambda(\mu \mathrm{m})$ & $n$ & $n \kappa$ & $n$ & $n \kappa$ & $n$ & $n \kappa$ \\
\hline 0,4358 & 1,56 & 0,46 & $\mathbf{1 , 5 7}$ & $\mathbf{0 , 4 6}$ & 1,57 & 0,46 \\
0,4500 & 1,56 & 0,48 & $\mathbf{1 , 5 6}$ & $\mathbf{0 , 4 9}$ & 1,56 & 0,50 \\
$\mathbf{0 , 5 3 2 0}$ & - & - & $\mathbf{1 , 5 7}$ & $\mathbf{0 , 5 0}$ & - & - \\
0,5500 & 1,56 & 0,46 & $\mathbf{1 , 5 7}$ & $\mathbf{0 , 5 0}$ & 1,57 & 0,53 \\
0,6500 & 1,57 & 0,44 & $\mathbf{1 , 5 6}$ & $\mathbf{0 , 4 9}$ & 1,56 & 0,52 \\
0,8065 & 1,57 & 0,46 & $\mathbf{1 , 5 7}$ & $\mathbf{0 , 4 8}$ & 1,57 & 0,49 \\
2,5 & 2,31 & 1,26 & $\mathbf{2 , 1 5}$ & $\mathbf{1 , 1 9}$ & 2,04 & 1,15 \\
3,0 & 2,62 & 1,62 & $\mathbf{2 , 3 7}$ & $\mathbf{1 , 3 9}$ & 2,21 & 1,23 \\
4,0 & 2,74 & 1,64 & $\mathbf{2 , 5 2}$ & $\mathbf{1 , 5 2}$ & 2,38 & 1,44 \\
5,0 & 2,88 & 1,82 & $\mathbf{2 , 3 9}$ & $\mathbf{1 , 7 6}$ & 2,07 & 1,72 \\
\hline
\end{tabular}

de suies par référence aux données spectrales fournies par Dalzell et Sarofim [6].

Au préalable, ces données doivent faire l'objet d'une double interpolation afin de :

- se ramener à la longueur d'onde utilisée dans la présente application;

- s'approcher des valeurs de $n$ et $n \kappa$ (cf. Éq. (6)) pour l'éthylène.

La première interpolation est justifiée par l'aspect continu du spectre attribué aux suies.

La seconde interpolation est, elle, plus délicate. En effet, les données de Dalzell et Sarofim ont été établies à partir de mesures de transmission à travers des suies de propane et d'acétylène. Or, la propension à la production de suies par une espèce combustible est intrinséquement liée à la fragilité de certaines de ses liaisons hydrocarbonées $\mathrm{C}-\mathrm{H}$. On corrèle donc fréquemment cette propension au rapport $\mathrm{H} / \mathrm{C}$ du nombre d'atomes d'hydrogène sur le nombre d'atomes de carbone. Fort heureusement, l'éthylène possède un rapport compris entre celui de l'acétylène et du propane. L'ensemble des interpolations est consigné en caractères gras dans le tableau 1 qui reporte en noir les données expérimentales de Dalzell et Sarofim.

La figure 9 est un exemple représentatif de la courbe de fraction volumique en suie le long de l'axe vertical $z$, déduite, par l'intermédiaire de l'équation (6), d'une évolution du coefficient d'extinction en $x=30 \mathrm{~mm}$.

\subsection{Estimation de l'épaisseur optique}

Comme le montre l'équation (3), une intégration dans la direction $z$ sur l'épaisseur de la couche de suie définit l'épaisseur optique à la longueur d'onde considérée. En revenant à l'équation (6), il est alors possible de connaître l'évolution de cette épaisseur optique le long du spectre du transfert thermique radiatif. La figure 10 est une illustration de cette évolution pour cet exemple didactique. 


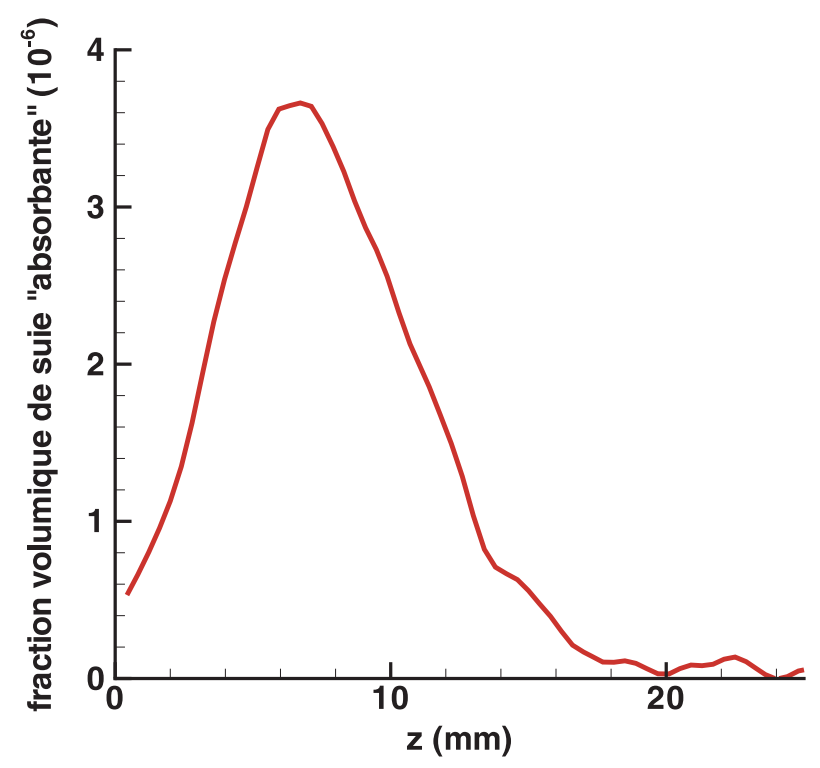

Fig. 9. Évolution de la fraction volumique de suies «absorbantes » en fonction de la coordonnée verticale $z$ pour $x=30 \mathrm{~mm}$.

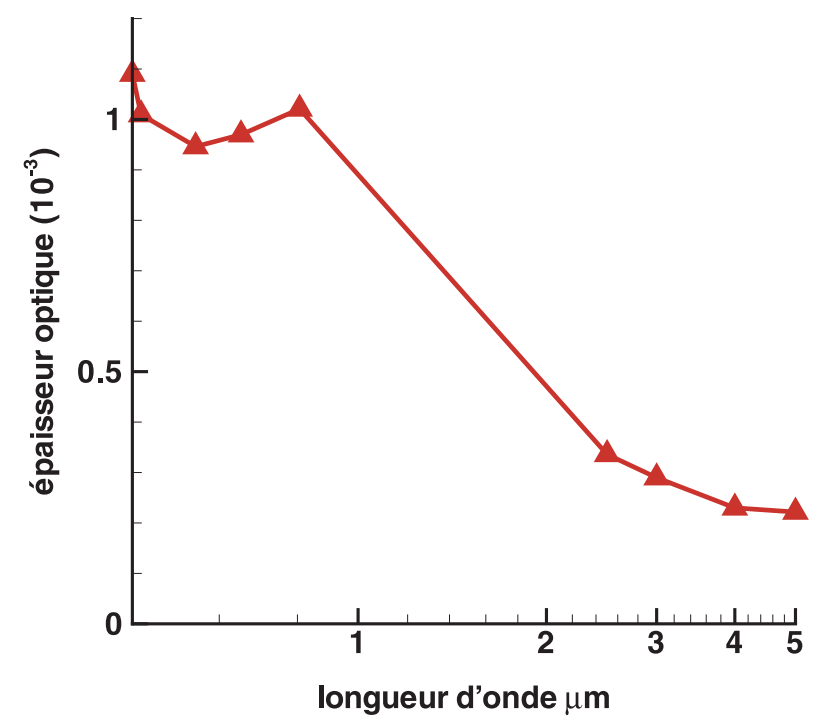

Fig. 10. Évolution le long du spectre du transfert radiatif de l'épaisseur optique de la suie.

\section{Conclusions}

Ce travail montre que dans le cas particulier exposé ici, la couche de suie ne peut absorber plus de $0.1 \%$ du spectre visible émis par la flamme et bien moins encore dans l'infra-rouge, où interviennent les bandes d'émission importantes du $\mathrm{CO}_{2}$ (particulièrement à $\lambda=2,7$ et $4,3 \mu \mathrm{m}$ ) et de $\mathrm{H}_{2} \mathrm{O}$ (en particulier à $\lambda=2,7$ et $6,3 \mu \mathrm{m}$ ). De fait, négliger l'absorption des suies dans cette flamme de diffusion établie en microgravité n'est pas déraisonnable, considérant toutefois uniquement la zone de stabilité définie dans cette étude.
Il reste à présent à considérer l'influence des paramètres d'injection et de soufflage sur la couche de suie ainsi que l'incertitude liée à la diffusion de flux radiatif par les suies.

\section{Références}

[1] NASA internal report NHB 8060, Flammability, Odor, Offgasing, and Compatibility Requirements and Test Procedures for Materials in Environments that Support Combustion (Office of Safety and Mission Quality), 1991

[2] J.L. Torero, T. Vietoris, G. Legros, P. Joulain, Estimation of a total mass transfer number from the stand-off distance of a spreading flame Combustion Science and Technology 174 (2002) 187-203

[3] H. Emmons, The Film Combustion ot Liquid Fuel, Z. Angews Math. Mech. 36 (1956) 60

[4] S. Rouvreau, P. Joulain, H.Y. Wang, P. Cordeiro, J.L. Torero, Numerical evaluation of boundary layer assumptions used for the prediction of the stand-off distance of a laminar diffusion flame, 29th Symposium (International) on Combustion, Sapporo, Japan, 2002

[5] R. Siegel, J.R. Hottel, Thermal Radiation Heat Transfer (second edition), Hemisphere Publishing Corporation, 1981

[6] W.H. Dalzell, A.F. Sarofim, Optical Constants of Soot and Their Application to heat-Flux Calculations, J. Heat Trans. 91 (1969) 100-104

[7] H.N. Najm, C.J. Mueller, P.S. Wyckoff, On the Adequacy of Certain Experimental Observables as Measurements of Flame Burning Rate, Comb. and Flame 113 (1998) 312-344

[8] K. Devriendt, H. van Look, B. Ceurters, J. Peeters, Kinetics of Formation of Chemiluminescent $\mathrm{CH}\left(A^{2} \Delta\right)$ by the elementary reactions of $\mathrm{C}_{2} \mathrm{H}\left(X^{2} \Sigma^{+}\right)$with $\mathrm{O}\left({ }^{3} P\right)$ and $\mathrm{O}_{2}\left(X^{3} \Sigma_{\mathrm{g}}^{-}\right)$: a pulse laser photolysis study, Chem. and Phys. Letter 261 (1996) 450-456

[9] B. Higgins, M.Q. McQuay, F. Lacas, S. Candel, An experimental study on the effect of pressure and strain rate on $\mathrm{CH}$ chemiluminescence of premixed fuel-lean methane/air flames, Fuel 80 (2001) 1583-1591

[10] P.A. Berg, D.A. Hill, A.R. Noble, G.P. Smith, J.B. Jeffries, D.R. Crosley, Absolute CH concentration measurements in low-pressure methane flames: Comparisons with model results, Comb. and Flame 121 (2000) 223-235

[11] M.G. Blevins, M.W. Renfro, K.H. Lyle, N.M. Laurendeau, J.P. Gore, Experimental Study of Temperature and $\mathrm{CH}$ Radical Location in Parially Premixed Coflow Flames $\mathrm{CH}_{4} / \mathrm{Air}$, Comb. and Flame 118 (1999) 684-696

[12] I.R. Hurle, R.B. Price, T.M. Sugden, A. Thomas, Sound emission form open turbulent premixed flames, Proc. Roy. Soc. (Lond.) A303 (1968) 409-427

[13] K. McManus, B. Yip, S. Candel, Emission and laserinduced fluorescence imaging in experimental combustion, Experimental thermal and fluid science 10 (1995) 486-502 